\title{
Chlamydia trachomatis genital infections
}

\author{
F. D. GHADIRIAN AND H. G. ROBSON \\ From the Division of Infectious Diseases, Departments of Medicine and Microbiology, Royal Victoria \\ Hospital and McGill University, Montreal, Quebec, Canada
}

SUMMARY The prevalence and clinical features of Chlamydia trachomatis infection were studied in men with nongonococcal urethritis (NGU), in their female sexual partners, and in other women. Of 92 patients with NGU, $36(39 \%)$ were chlamydia-positive; although all had symptoms of urethritis, fewer than half had evident discharge. Clinical features of chlamydia-positive and chlamydia-negative NGU were indistinguishable. Most female contacts of chlamydia-positive patients with NGU were infected with $C$. trachomatis; contacts of chlamydia-negative patients with NGU seldom harboured chlamydia. Inflammatory and erosive changes were equally common in the uterine cervix of chlamydia-positive and chlamydia-negative women, although almost half of infected cases had no clinical signs of infection and no specific symptoms. In such women the present unavailability of sensitive screening techniques is a major obstacle to the effective control of chlamydial infection.

\section{Introduction}

Increasing emphasis is being placed on the role of Chlamydia trachomatis as a human pathogen, especially in infection of the genital tract. In men, $C$. trachomatis has been identified as an important cause of nongonococcal urethritis (NGU) (Dunlop et al., 1972; Holmes et al., 1975) and of ascending genital infections such as epididymitis (Harnisch et al., 1977).

Although $C$. trachomatis has been isolated with increased frequency from the cervix of female sexual partners of men with chlamydia-positive NGU (Oriel et al., 1972; Holmes et al., 1975), its role as, a pathogen in women is less clearly defined. Recent studies have provided evidence that infection may lead to cervical abnormalities (Swanson et al., 1975; Rees et al., 1977; Oriel et al., 1978) and to salpingitis (Mårdh et al., 1977).

Nongonococcal urethritis is not a notifiable disease in our community and there are no accurate statistics as to its prevalence. Experience in our clinic suggests that the disease occurs at least as often as gonococcal urethritis. The present study was undertaken to determine the prevalence of $\boldsymbol{C}$. trachomatis infection in men attending our outpatient clinic with symptoms of nongonococcal urethritis, in their female contacts, and in women who presented for examination to exclude sexually transmitted diseases. We also

Address for reprints: Dr H. G. Robson, 3775 University Strecl, Montreal, Quebec H3A 2B4, Canada attempted to define the symptoms and abnormal clinical findings of our patients in relation to chlamydial isolation.

\section{Patients and methods}

\section{SELECTION OF CASES}

The study population was composed of patients who attended the Infectious Disease Clinic of the Royal Victoria Hospital between 18 November 1977 and 2 May 1978. The male patients included in the study were those with complaints of urethral discharge or dysuria but with negative results to Gram-stained urethral smears and to cultures for Neisseria gonorrhoeae on presentation at the clinic. Some men had been treated for culture-positive gonococcal urethritis within the preceding 28 days. Female sexual partners of some of the male patients in the study were included as were some women who attended clinic for screening examinations.

Chlamydial isolations were attempted only from patients who had not received antibiotic therapy within the preceding four weeks, except for penicillin treatment of gonorrhoea. The latter patients had been treated with single-dose regimens of aqueous procaine penicillin $G \mathbf{4} \cdot 8$ megaunits intramuscularly or ampicillin $3.5 \mathrm{~g}$ orally, each with probenecid $1 \mathrm{~g}$ orally.

\section{CLINICAL EVALUATION}

A standard medical history was obtained from each patient; in addition information on sexual behaviour 
and previous episodes of sexually transmitted disease was requested. Examination of male genitals included inspection of the penis and inguinal regions as well as gentle milking of the urethra in those patients who had no frank discharge. In most cases a two-glass urine test was performed. The criteria of Holmes et al. (1975) for diagnosing NGU were used: the presence of pyuria in the first $10 \mathrm{ml}$ of voided urine, and the presence of 20 or more leucocytes in at least one of five randomly chosen microscopic fields at $\times \mathbf{4 0 0}$ magnification.

Female patients underwent genital examination which included inspection of the cervix after removal of adherent secretions. Abnormalities such as the presence of vaginal discharge, cervical erosions, inflamed or friable cervical epithelium, and hypertrophic nodular or cystic changes in the epithelium surrounding the cervical os were recorded.

\section{COLLECTION OF SPECIMENS}

Material for isolation of $C$. trachomatis was obtained with calcium-alginate swabs passed into the anterior urethra of men or endocervical canal of women. Swab tips were cut into plastic vials containing 2SPS transport medium (Darougar et al., 1972). This material was kept at $4^{\circ} \mathrm{C}$ for up to four hours after collection and then stored at $-70^{\circ} \mathrm{C}$ until inoculation into cell culture. Specimens for culture for $N$. gonorrhoeae were also obtained from the male urethra and from the cervix and rectum of female patients.

\section{CELL CULTURE}

The McCoy cell line was kindly provided by Dr S. Darougar (Institute of Ophthalmology, London). Confluent monolayers of cells in tissue culture flasks were trypsinised and suspended in CMA (Darougar et al., 1971). One millilitre of cell suspension adjusted to contain $1 \times 10^{5}$ cells $/ \mathrm{ml}$ was added to each of a series of flat-bottomed tubes containing a 13-mm diameter coverslip. After 48-72 hours' further incubation at $35^{\circ} \mathrm{C}$ confluent monolayers were ready for inoculation of specimens.

\section{ISOLATION OF C. TRACHOMATIS}

Specimens were rapidly thawed, agitated vigorously, diluted 1/2 with CMGA (Gordon et al., 1969), and then inoculated in duplicate in flat-bottomed tubes from which the CMA had been removed. The inoculated tubes were centrifuged at $15000 \times g$ for one hour at $35^{\circ} \mathrm{C}$. After a further two hours' incubation at $35^{\circ} \mathrm{C}$ the inoculum was replaced with fresh CMGA containing cycloheximide $1 \mu \mathrm{g} / \mathrm{ml}$ and the tubes were incubated for a further 72 hours. At that time the coverslips were Giemsa-stained and examined by darkfield microscopy at $\times 200$ magnification for the presence of characteristic inclusions.

\section{STATISTICS}

Yates's $\chi^{2}$ test was used for statistical evaluations.

\section{Results}

\section{DEMOGRAPHIC AND CLINICAL FINDINGS}

Men with NGU

Ninety-two male patients were included in the study. Of these, $36(39 \%)$ were found to be infected with $C$. trachomatis. No features either in the history or on clinical examination were found to be useful in distinguishing culture-positive from culture-negative patients (Table 1). Urethral discharge could be

Table 1 Demographic and clinical features in male patients with NGU

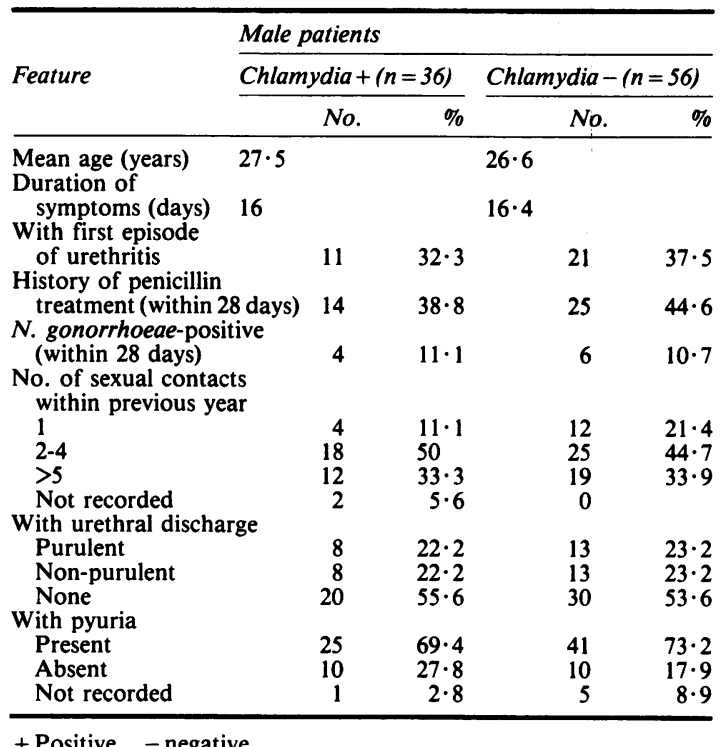

objectively documented in $44 \%$ of chlamydiapositive and of $46 \%$ of chlamydia-negative patients. When present, the urethral discharge was equally often described as purulent or non-purulent. Although most patients had pyuria, $28 \%$ of men with positive culture results had negative results to the two-glass urine test. Thirty-two and $38 \%$ of culturepositive and culture-negative patients respectively stated that they had not previously had urethritis. The age of the patients, duration of their symptoms, number of sexual contacts in the preceding year, and 
history of penicillin treatment within the preceding 28 days did not differ significantly between the two groups. Two $(5 \cdot 5 \%)$ of 36 chlamydia-positive patients and seven $(12 \cdot 5 \%)$ of 56 culture-negative patients claimed to engage solely in homosexual relationships.

\section{Female patients}

Eighteen of $\mathbf{4 5}$ women studied were infected with $C$. trachomatis (Table 2). The mean age of those infected was similar to that of the culture-negative group. Certain features such as the use of oral contraceptives, more than one sexual partner in the preceding year, and a concurrently positive culture result for $N$. gonorrhoeae appeared to be more frequent in the chlamydia-positive group; however none of the differences was statistically significant ( $P>0.05$ in all instances).

Table 2 Demographic features in female patients with and without chlamydial infection

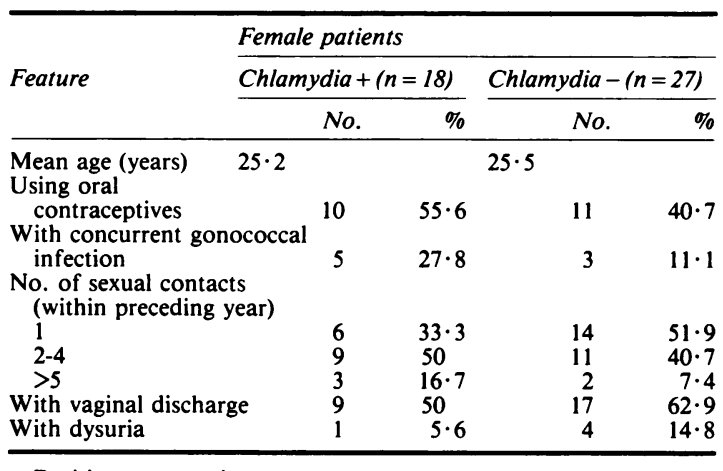

+ Positive - negative

Cervical abnormalities were detected in both chlamydia-positive and chlamydia-negative patients (Table 3). The presence of inflammation, erosion, nodular or cystic changes, and friability of the cervical epithelium did not differ significantly between the two groups. It was striking that nearly half $(\mathbf{4 4 \%})$ of patients shown to be infected with $C$. trachomatis had an entirely healthy cervix on clinical examination.

\section{Female partners of infected men}

We obtained specimens for culture from 11 contacts of men known to be infected with $C$. trachomatis (Table 4). Of these women, seven were chlamydiapositive while two others were found retrospectively to have taken a course of tetracycline within the preceding month. Of 10 contacts of chlamydianegative men, only one was infected. This difference was significant $\left(\chi_{1}^{2}=4 \cdot 80, P<0 \cdot 05\right)$.
Table 3 Abnormalities of cervix in relation to chlamydial infection

\begin{tabular}{|c|c|c|c|c|}
\hline \multirow{3}{*}{ Abnormality } & \multicolumn{4}{|c|}{ Female patients } \\
\hline & \multicolumn{2}{|c|}{ Chlamydia $+(n=18)$} & \multicolumn{2}{|c|}{ Chlamydia $-(n=27)$} \\
\hline & No. & $\%$ & No. & $\%$ \\
\hline \multirow{2}{*}{$\begin{array}{l}\text { None } \\
\text { Inflammation } \\
\text { Erosion } \\
\text { Friability } \\
\text { Nodular or cystic } \\
\text { changes }\end{array}$} & $\begin{array}{l}8 \\
7 \\
4 \\
6\end{array}$ & $\begin{array}{l}44 \cdot 4 \\
38 \cdot 9 \\
22 \cdot 2 \\
33 \cdot 3\end{array}$ & $\begin{array}{r}13 \\
10 \\
4 \\
5\end{array}$ & $\begin{array}{l}48 \cdot 1 \\
37 \cdot 0 \\
14 \cdot 8 \\
18 \cdot 5\end{array}$ \\
\hline & 3 & $16 \cdot 7$ & 3 & $11 \cdot 1$ \\
\hline
\end{tabular}

Table 4 Chlamydial infection in female contacts of men with NGU

\begin{tabular}{llll}
\hline & & \multicolumn{2}{l}{ Female contacts } \\
\cline { 3 - 4 } Male patients & No. & No. examined & No. chlamydia + \\
\hline Chlamydia-positive & 36 & $11^{*}$ & 7 \\
Chlamydia-negative & 56 & 10 & 1 \\
\hline
\end{tabular}

+ Positive

* Two patients had previously received tetracycline therapy

\section{Discussion}

The isolation rate of $39 \%$ for C. trachomatis from men with NGU is in agreement with the results of many other studies (Dunlop et al., 1972; Oriel et al., 1972; Holmes et al., 1975; Schacter et al., 1975). Some have reported isolation rates of over $50 \%$ (Perroud and Miedzybrodzka, 1978; Terho, 1978). Our patients were relatively unselected; we required only that they had complained of dysuria and urethral discharge and had negative culture results for $N$. gonorrhoeae. Since our clinic operated on only two mornings a week we found it impractical to recall patients for repeat examinations and cultures after overnight urinary continence. This may be the reason we could not record the presence of urethral discharge on clinical examination in most (50/92) patients in the study, and may explain why pyuria was absent in the first-glass urine of 10 of 36 chlamydia-positive men. Examining patients under less than ideal conditions did not appear to impair our ability to recover chlamydia from their urethral cultures.

Several authors have shown a correlation between the severity of urethral discharge and an increased isolation rate for $C$. trachomatis (Schachter et al., 1975; Alani et al., 1977; Terho, 1978). This correlation was not observed in our patients, as $16 / 42(38 \%)$ with, and $20 / 50(40 \%)$ without, visible discharge were chlamydia-positive. Furthermore, we were unable to confirm any relationship between the duration of symptoms and the rate of recovery of $C$. 
trachomatis described by Holmes et al. (1975) and Alani et al. (1977).

Retrospective analysis of the histories and clinical findings in our male patients showed no clinically important differences between those with NGU who were infected with $C$. trachomatis and those who were not, as shown in several other studies (Oriel $e t$ al., 1972; Holmes et al., 1975; Perroud and Miedzybrodzka, 1978). This also poses a serious problem in the assessment of female patients. Numerous authors have described abnormalities of the uterine cervix in chlamydia-positive patients such as erosions, follicular and cystic lesions, and purulent cervical secretions (Dunlop et al., 1972; Hilton et al., 1974; Oriel et al., 1974). Rees et al. (1977) have stated that hypertrophic cervical erosions and mucopurulent cervical discharge occur with increased frequency in chlamydia-positive patients. However, such abnormalities are not specific to chlamydial infection but may also be caused by Trichomonas vaginalis (Oriel et al., 1978) and possibly by other agents. Although the number of female patients studied by us was small we could not distinguish on clinical grounds those who were infected with $C$. trachomatis from those who were not. Moreover, we found that many of our chlamydia-positive female patients had no complaints and appeared to be entirely healthy on clinical examination. This finding is certainly not unique to our study but again underlines the existence of a large infectious pool which is difficult to detect and is capable of perpetuating infection in the population. The application of serological procedures for population screening as described by Treharne et al., (1978) may increase our ability to detect and control infection by C. trachomatis.

\section{References}

Alani, M. D., Darougar, S., Burns, D. C. Mac D., Thin, R. N., and Dunn, H. (1977). Isolation of Chlamydia trachomatis from the male urethra. British Journal of Venereal Diseases, 53, 88-92.
Darougar, S., Kinnison, J. R., and Jones, B. R. (1971). Simplified irradiated McCoy cell culture for isolation of chlamydiae. In Trachoma and Related Disorders, p. 63. Edited by R. L. Nichols, Excerpta Medica: Amsterdam.

Darougar, S., Jones, B. R., Kinnison, J. R., Vaughan-Jackson, J. D., and Dunlop, E. M. C. (1972). Chlamydial infection. Advances in the diagnostic isolation of Chlamydia, including TRIC agent, from the eye, genital tract, and rectum. British Journal of Venereal Diseases, 48, 416-420.

Dunlop, E. M. C., Vaughan-Jackson, J. D., Darougar, S., and Jones, B. R. (1972). Chlamydial infection. Incidence in "nonspecific' urethritis. British Journal of Venereal Diseases, 48, 425-428.

Gordon, F. B., Harper, I. A., Quan, A. L., Treharne, J. D., Dwyer, R. St. C., and Garland, J. A. (1969). Detection of Chlamydia (Bedsonia) in certain infections of man. I. Laboratory procedures: comparison of yolk sac and cell culture for detection and isolation. Journal of Infectious Diseases, 120, 451-462.

Harnisch, J. P., Berger, R. E., Alexander, E. R., Monda, G., and Holmes, K. K. (1977). Aetiology of acute epididymitis. Lancet, 1, 819-821.

Hilton, A. L., Richmond, S. J., Milne, J. D., Hindley, F., and Clarke, S. K. R. (1974). Chlamydia A in the female genital tract. British Journal of Venereal Diseases, 50, 1-10.

Holmes, K. K., Handsfield, H. H., Wang, S. P., Wentworth, B. B., Turck, M., Anderson, J. B., and Alexander, E. R. (1975). Etiology of nongonococcal urethritis. New England Journal of Medicine, 292, 1199-1205.

Mårdh, P-A., Ripa, T., Svensson, L., and Westrom, L. (1977). Chlamydia trachomatis infection in patients with acute salpingitis. New England Journal of Medicine, 296, 1377-1379.

Oriel, J. D., Johnson, A. L., Barlow, D., Thomas, B. J., Nayyar, K., and Reeve, P. (1978). Infection of the uterine cervix with Chlamydia trachomatis. Journal of Infectious Diseases, 137, 443-451.

Oriel, J. D., Powis, P. A., Reeve, P., Miller, A., and Nicol. C. S. (1974). Chlamydial infections of the cervix. British Journal of Venereal Diseases, 50, 11-16.

Oriel, J. D., Reeve, P., Powis, P., Miller, A., and Nicol. C. S. (1972). Chlamydial infection. Isolation of Chlamydia from patients with non-specific genital infection. British Journal of Venereal Diseases, 48, 429-436.

Perroud, H. M. and Miedzybrodzka, K. (1978). Chlamydial infection of the urethra in men. British Journal of Venereal Diseases, 54, 45-49.

Rees, E., Tait, I. A., Hobson, D., and Johnson, F. W. A. (1977). Chlamydia in relation to cervical infection and pelvic inflammatory disease. In Nongonococcal urethritis and related infections, pp. 67-76. Edited by D. Hobson and K. K. Holmes. American Society for Microbiology: Washington DC.

Schachter, J., Hanna, L., Hill, E. C., Massad, S., Sheppard, C. W., Conte, J. E. Jr., Cohen, S. N., Meyer, K. F. (1975). Are chlamydial infections the most prevalent venereal disease? Journal of the American Medical Association, 231, 1252-1255.

Swanson, J., Eschenbach, D. A., Alexander, E. R., and Holmes, K. K. (1975). Light and electron microscopic study of Chlamydia trachomatis infection of the uterine cervix. Journal of Infectious Diseases, 131, 678-687.

Terho, P. (1978). Chlamydia trachomatis in non-specific urethritis. British Journal of Venereal Diseases, 54, 251-256.

Treharne, J. D., Darougar, S., Simmons, P. D., and Thin, R. N. (1978). Rapid diagnosis of chlamydial infection of the cervix. British Journal of Venereal Diseases, 54, 403-408. 